\title{
An Efficient Rule based Decision Support System using Semantic Web Technology
}

\author{
Jawed Naseem ${ }^{1}$ \\ Statistical Support and IT Unit \\ Pakistan Agricultural Research \\ Council Karachi, Pakistan
}

\author{
S. M. Aqil Burney ${ }^{2}$ \\ College of Computer Science \\ IOBM University \\ Karachi, Pakistan
}

\author{
Nadeem Mehmood ${ }^{3}$ \\ Department of Computer Science \\ University of Karachi \\ Karachi, Pakistan
}

\begin{abstract}
The Semantic Web technology is an efficient mechanism to query or infer knowledge on a global scale using the internet by providing logical reasoning through rule based system. In this paper application of semantic web technology is discussed in context of agriculture knowledge management and delivery. In agriculture, adoption of newly developed technology is essential to enhance crop production. However, timely dissemination of authenticated agriculture information for decision making at larger scale to diversified end user has always been a challenge due to several reasons. One of the reasons is storing and delivering agriculture knowledge in machine readable form. In this paper a frame work based on semantic web is presented for collection, storing and updating of agricultural information at centralized location and delivering knowledge through intelligent decision support system through semantic web. The frame work utilizes rule based system for querying information from agriculture knowledge base.
\end{abstract}

Keywords-Agriculture information; semantic web; rule based system; intelligent DSS

\section{INTRODUCTION}

Agricultural research ensures food security of a nation. Developing agricultural technology and its dissemination to end user is the only option for sustainable agriculture development. The timely communication of newly developed research based technology to end-user for adoption is a challenge. The wider gap is always existed between development of technology and its adoption at farm level due to absence of efficient mechanism for delivery of information to end-user [9]. The conventional method of agricultural extension supported by use of information technology can fill the wider gap for access to agricultural information.

Crop production management is an essential component in agriculture. Monitoring of several activities are required during plant growth to ensure appropriate development of plant and maximize yield. Decision involved are planning pertaining to pre cultivation (selection of varieties, land preparation etc) monitoring during cultivation \& growth (irrigation management, fertilizer application) and post harvest management. Feasible decision making requires access to latest relevant information to use appropriate technology, methods. Secondly, processing information with expert perspective and propose solution or action for end-user.

Presently computer science and information technology provide enormous opportunities for dissemination of information in every field of life [1]. From a standalone computer application or database on a machine to an organizational network or World Wide Web information can be process and disseminated quickly and efficiently. Several types of DSS has been developed in agriculture [7] and [11]. An ontology based domain rule development has been discussed by [8]. An intelligent plant disease diagnoses system has been presented by [10] and [11].

The ontology based knowledge engineering in agriculture is discussed by [2]. Burney has proposed a Bayesian network for wheat disease diagnoses in production management [12].

The utilization of semantic web for decision support has relevance particularly in agriculture. The Semantic Web shares many goals with Decision Support Systems (DSS) mainly to interpret information precisely to deliver relevant reliable and accurate information to a end user irrespective of time and location. While DSS have more specific goals, since the information need is targeted towards making a particular decision, e.g., selecting a particular crop variety for improved yield

In this research a case study of knowledge management of wheat production technology in Pakistan is under taken. A frame work and rule based system is presented for decision making in wheat production management through semantic web. Section I contain introduction, Section II discuss challenges and issues in wheat production management, Section III discuss semantic web technology, Section IV present an efficient rule based systems for framing queries and retrieval information through semantic web. Conclusion is discussed in Section V

\section{DYNAMICS IN DISSEMINATION OF AGRICULTURE TECHNOLOGY}

Sustainable agricultural development requires adaptation and incorporation of new technologies developed to enhance agricultural production. The technology may involve development of new varieties, agricultural production management, water management or crop protection. Adaptation of these technologies involves continuous updating of knowledge regarding a particular technology and processing of information by expert to deliver appropriate solution to problem in agriculture. High level human expertise in agriculture, like other disciplines of science, are not only scares but also costly. Beside this expert knowledge in 
agriculture require mass dissemination of information to large audience of end-users including policy makers, researcher, extension people and ultimately to farmer. Conventional means of communication of agricultural information have limited scope of knowledge acquisition, processing and instant delivery to end-user. Computer based systems [7] and soft computing models can provide an effective and efficient knowledge management [3]. Knowledge engineering in domain of agriculture comprises three basic component, Knowledge acquisition \& representation, information processing and delivering of possible solution.

In a system where a number of experts contribute to knowledge base it is challenging to develop a consensus on various options. Therefore developing a mechanism for consensus of expert is essential in knowledge base system. [1] has proposed a system to increase group consensus which is based on consistency as well as measuring consensus together with maintaining the individual opinion of each expert.

\section{A. Spatio Temporal Variation}

Spatio temporal variation is one of the challenge in development of DSS in agricultural. Wheat production technology significantly varies across the country based on agricultural zones which are classified on the basis of environmental parameter. Research system has developed wheat varieties which are suitable to particular zone. The variation not only affects wheat quality and yield but risk associated with the attack of diseases and pest of wheat crop. Particular disease and pest are more vulnerable in particular environment. Like intensity of pest reduces by increase of temperature. Beside this within agricultural zone (spatial variation) temporal variation also come into focus. Yield of wheat is affected by the planting date of wheat variety. With late sowing of crop yield is reduced over time. Developing a computing model which captures this spatio temporal variation is essential.

\section{B. Uncertainty}

Crop growth in agriculture encompasses several uncertain situation, weather condition, timely access to agricultural input, spread of disease $\&$ pest and scheduling of competing crop. Predicting the uncertain outcome is essential for timely decision making in wheat growth. Whether timely harvest of previous crop will affect planting date of wheat? How temperature and humidity over next week will favor growth of pest? Whether required fertilizer will be available in market for timely application? These are the question which requires accurate answer (as much as possible) for sustainable growth of wheat. To some extend wheat yield is a game of chance also. The combination any of these uncertain situations can lead to make or break scenario of wheat crop. It is not very uncommon that uncertain weather condition may destroy wheat crop completely. No soft computing model for management of crop can succeeds without capturing inherent uncertainty in affecting factors. Luckily many methods in AI are capable of predicting uncertainty.

\section{Knowledge Representation}

Agriculture like any other discipline is huge canvas of knowledge comprising thousands of concepts and hundreds of relationships between those concepts. Representing knowledge in machine readable form is the basic element in developing computing system. However, agriculture is different in the sense that it contain common simple to understand concepts like suitable varieties along with highly technical information like agronomy, pathology and entomology etc. Agriculture had very varied audience; on one end are the farmers mostly accustomed with conventional terminology in local language and on the other end is sophisticated scientific community of researcher who are supposed to develop or update agriculture knowledge. Developing a common frame work which is capable of this varied semantic level of the audience is real challenge which is successfully addressed in this research. Development of Owl based ontology [13] is an option for knowledge representation in agriculture. The advantage of OWL base ontology is the fact that owl and RDF are effective tools of semantic web [6].

\section{SEMANTIC WEB}

The Semantic Web is the latest development for real time sharing of information on internet. Semantic web facilitate a common mechanism which allows sharing of data across different application, enterprise, and community. Semantic web expresses information in machine-readable form. It is the web that can be processed by machines in such a way that meaning of different concepts is commonly expressed. Technically semantic web is a giant data graph defined in RDF.

The common technology used in semantic web is OWL (Web Ontology Language) ontology expressed in RDF graph. OWL is a Semantic Web language designed to express knowledge about concepts and relations between concepts [13]. OWL is a logic-based computing language which may be used by computer systems. OWL ontology can be published in the World Wide Web and may refer to other ontology or other ontology use it [15]. The semantic web includes a stack of WC3 technology comprising OWL RDF, RDFS, SPARQL, etc. Basically Web Ontology Language (OWL) is a modeling language encompasses fast and flexible data modeling and efficient automated reasoning. OWL extends RDF (Resource Description Framework) and RDFS.

The semantic web use graph data model to store information and RDF is the format in which this information is written. The information contained in a Web resource is expressed in XML-based language using RDF for description. The resource can be knowledge base, a Web page, an entire Web site, or any item on the Web that contains information in some form. RDF can be used to define any of the resources. However in case of knowledge base or representation through ontology the resource may be a URI (uniform resources identifier). RDF is a tool of meta data description used for encoding, exchange, and reuse of structured data. The use of RDF is domain independent it has not generalized application without assumption about any particular domain, nor semantics is depended on domain.

The RDF [14] graph can be queried for knowledge search. SPARQL Protocol and RDF Query Language (SPARQL) is the language to query a RDF graph. Its syntax is almost 
similar to SQL the main difference is SQL can query relational database while SPARQL can query NOSQL or database graph. In fact a SPARQL query can also be executed on any database that can be viewed as RDF via middleware. For instance, a relational database can be queried with SPARQL by converting Relational Database to RDF. SPARQL is capable of querying diverse data source no matter whether data is expressed in RDF or in a form which can be converted in to RDF both can be queried through SPARQL. SPARQL have capability to query several graph pattern using conjunction or disjunction attribute of the graph. The output of SPARQL queries can be results sets or another RDF graphs.

\section{Frame Work FOR SEMANTIC WEB WheAT PRODUCTION TECHNOLOGY}

In agriculture knowledge generation is scattered over many sources and by many roles. Traditionally in Pakistan agricultural knowledge is disseminated through conventional means through extension department. Agriculture Knowledge generator (researcher) has limited access to disseminator (extension worker) or vice versa. Secondly, communication between extension worker and ultimate user (farmer) is fragile. This wider gap can be filled only through unconventional means. A central knowledge base accessible to all stake holders can be achieved through web based technologies where authorized user with defined roles are provided with tools to update the required information. Centralized web based knowledge management and updating ensure timely availability of authenticated information.

We have suggested a frame work (Fig 1) for development of collaborative updating of knowledge to central repository by a panel of authorized person from research and non research establishment using common electronic tools of semantic web. This frame work not only ensures data authentication but remove data redundancy.

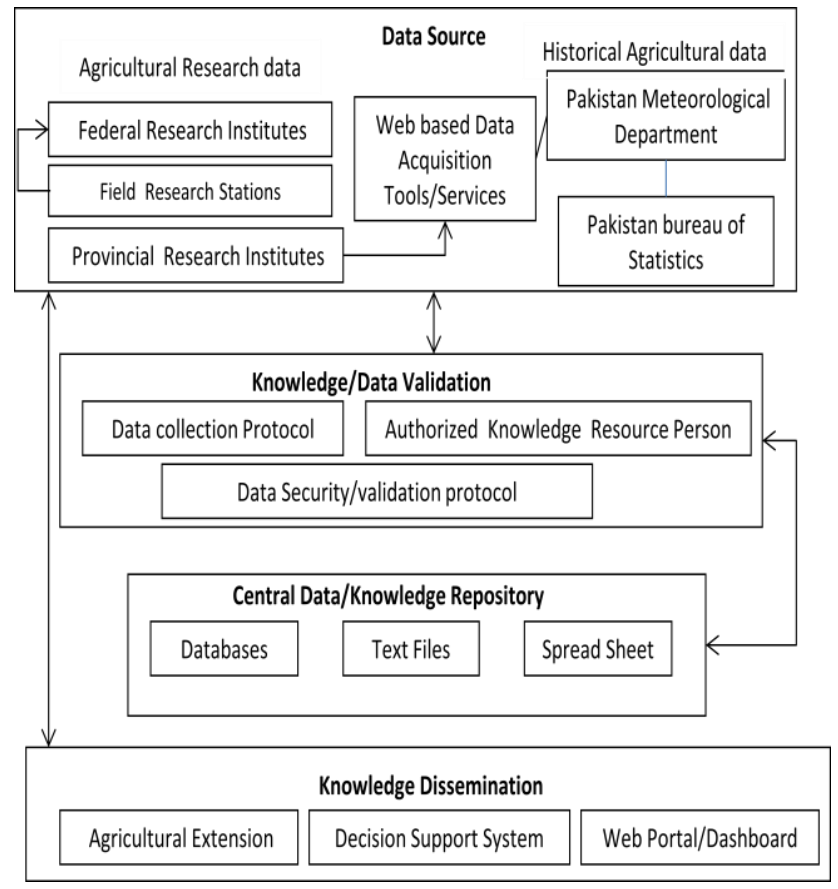

Fig. 1. Frame Work for Semantic Web of Wheat Production Technology.

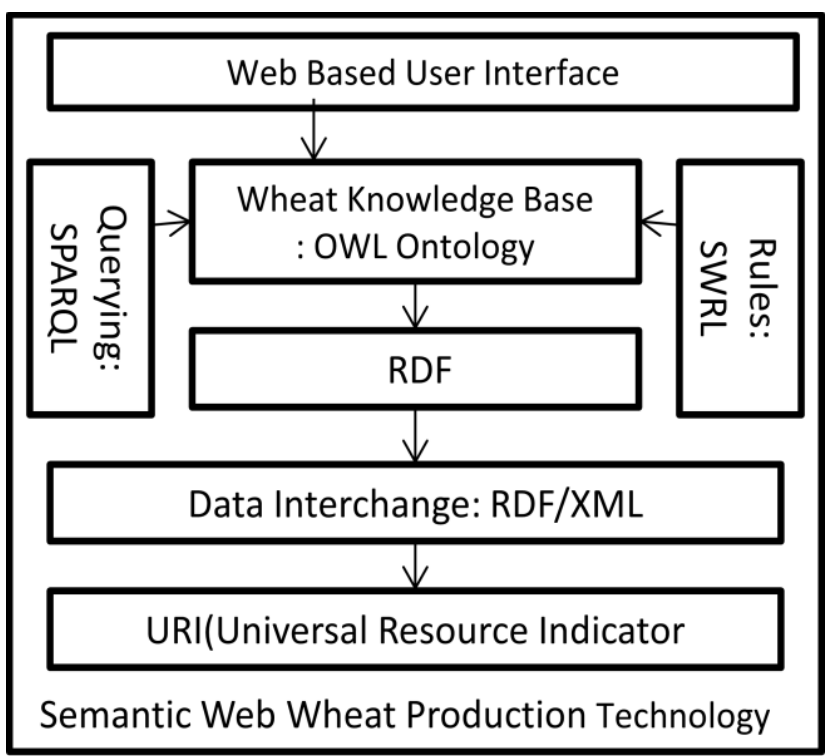

Fig. 2. Semantic Web Wheat Production Technology.

The second step of this process is mapping of the suggested frame work into a machine readable form to create a central knowledge repository which is achieved through semantic web (Fig 2). The frame work is used to collect information from relevant research institutes and department which may in the shape of databases, flat text file or other medium.

The semantic web comprises knowledge base development using owl ontology and mapping it through RDF [4]. Reasoning the KB using WSRL [5] rules or SPARQL query [14] and delivering the information through URI. On the top of it is the web based user interface to interact with the end user.

The user formulate query using interface and solution to problem is communicated by identifying relevant URI in the knowledgebase.

\section{RULES BASED SYSTEM FOR QUERYING SEMANTIC WEB}

Rules based expert system and rules are structured way of reasoning and classifying information. Production rules in the form of if \& then clause are used to define or apply constrain in declarative manner. Domain rules are structured in informal, semi informal and formal ways. However, in knowledge base rules are expressed in formal system. Informal statements in natural language are transformed into formal language or rule execution language.

In semantic web rules can be developed by using Semantic Web Rule Language (SWRL)[5]. SWRL represent rules as well as define logic of extraction by combining OWL DL with the Rule Markup Language.

SWRL develop Horn-like rules by extending the set of OWL axioms which represent logical extraction from knowledge base. SWRL follow traditional rule construction in the form of an implication between an antecedent (body) and consequent (head). So SWRL rule can be read as: whenever the conditions declared in the antecedent are true, then the 
conditions specified in the consequent must also be true. In OWL ontology abstract syntax contains a sequence of axioms and facts. Each Axiom may be of different kinds, e.g., subClass axioms or equivalent Class axioms. Then axioms are extended to rule.

axiom::= rule

A rule based on axiom consists of an antecedent (body) and a consequent (head), each of which consists of a set of atoms which may be possibly empty. Therefore, a URI reference can be assigned in axiom which could serve to identify the rule.

Rule:: = 'Implies(' [URIreference ] \{ annotation $\}$ antecedent consequent ')'

antecedent::= 'Antecedent(' \{ atom \} ')

consequent::= 'Consequent(' \{ atom \} ')

Where both antecedent and consequent can be conjunctions of atoms written in the form

a $1 \wedge \ldots$ a $2 \wedge$

While variables are indicated by using a question mark (e.g., ?x). The advantage of SWRL rule is it can be represented in human readable form. In the abstract syntax the rule would be written like

Implies(Antecedent(Property1(I-variable(x1)IVariable(x2))

Property2 (I-variable(x2) I-variable(x3)))

Consequent(Property3(I-variable(x1)I-variable(x3))))

The following rule describes selection of agricultural zone on the basis of district and province. When district is $y$ and province is $\mathrm{x}$ agricultural zone is $\mathrm{Z}$

District (?y)^ province(?x) -------> zone(?z)

Where LHS represent antecedent and RHS represent consequent. It may be noted that antecedent is conjunction of atom using and properties while consequent is atomic.

In AI several methods can be used for formal expression or rules like SQL (Structured Query Language), ECA, predicate logic and propositional logic. In wheat production expert system ontology based predicate logic and axioms are used to define rules for extraction and updating of information from wheat knowledge base. Developing ontology based rule is three steps process [8] involving

- Express rule in informal natural language

- Express rule using ontology concepts and relationship

- Express rule in formal predicate logic

In wheat production management expert system conditions and actions are proposed by agriculture expert in natural language which is expressed into ontological expression and finally into SWRL syntax

Informal: if soil is loamy and soil condition is weak then apply 3 bag of NPK or 2 bag of DAP at the time of sowing.
Using ontological expression rule can be expressed as

$$
\begin{aligned}
& <\text { Soil }><\text { has_type }><\text { loamy }>\text { and } \\
& <\text { Soil }><\text { has_condition }><\text { Weak }> \\
& \text { then }<\text { Fertilizer }><\text { has_name }>=\text { "NPK" }<\text { hasQuantity }>= \\
& \text { "2 bags", Or } \\
& \text { Fertilizer }><\text { has_name }>=\text { "DAP" }<\text { hasQuantity }>=\text { "3 } \\
& \text { bags", } \\
& \text { and }<\text { Fertilizer }><\text { hastimeof application }>=\text { "at sowing" }
\end{aligned}
$$

The ontological expression is transformed into rule using SWRL syntax. So formal expression in predicate logic will be

SoilType(?x) ^ SoilCondition(?y) $---\rightarrow$ Fertilizer(?z)

Fertilizer(?f) $\wedge$ hastimeofapplication(?t) --- $\rightarrow$ has_quantity(?q)

The terms in bracket represent variable which are replaced by named individual. The SWRL rules based knowledge can be queried using DL or SPARQL query. SPARQL query has sql like syntax as follows

SELECT ?subject ?object

WHERE \{ ?subject rdfs:subClassOf ?object \}

So using the query syntax quantity of fertilizer for weak soil can be retrieved using following query

\section{SELECT ?Fertilizer ?quantity}

WHERE \{ soil ? has_condition? weak \}

The three step process seamlessly transform parameter based question into to appropriate machine readable rule which extract required information from knowledgebase and delivered it through relevant semantic web URI

\section{CONCLUSION AND DISCUSSION}

This paper presents an intelligent DSS based on semantic web for facilitating the decision making in wheat production management. A frame work is proposed for deployment of DSS through semantic web. Effective decision support is provided with the development of ontology driven rule based system by considering both the characteristic of the problem and the requirements of the decision maker.

The applicability of the frame work is demonstrated through the example of decision support for wheat production management. The example shows that the proposed DSS framework has a number of advantages over data driven desktop based DSS. The proposed DSS has three specific advantage first its ensure wider dissemination through internet, secondly it represent knowledge in logical way by using ontology, thirdly it is intelligent. The DSS is intelligent in the sense that it not only effectively retrieves required information by efficient query but also infer the knowledge which is not physically present in the knowledge base by extending logical reasoning of an expert through build in rule based system. In domain of agriculture updating of knowledge at wider scale through many sources is equally essential as retrieving of information. The ontology based semantic web resolve this issue effectively by providing a mechanism following logical updating and resolving conflicting information at real time. The knowledge is not updated until it meets required protocol. 
Application of semantic web in agriculture is particularly become more relevant as generation of knowledge is scattered over many sources so updating of knowledge become more feasible with minimum IT resources available which is one of the main constraint in under developing countries. This paper proposes a system which can be implemented economically at wider scale. The proposed frame work ensures scalability and interoperability as it can be modified for other crops also.

The proposed system has one limitation that, as the new knowledge is generated obsolete information should be discarded automatically as removal of outdated information is essentially important. The next research topic related to this study is to develop an automated process which can periodically identify obsolete information, validate in context of new information and update it accordingly.

\section{REFRENCES}

[1] Alonso S., E. Herrera-Viedmab, F. Chiclanac, F. Herrerab, 2010, “A web based consensus support system for group decision making problems and incomplete preferences" Information Sciences, Volume 180, Issue 23,

[2] Burney, S. M Aqil, Jawed Naseem, 2018," Knowledge Engineering in Agriculture: A Case Study of Soft Computing Model for Wheat Production Management", International Journal of Computer Science and Information Security (IJCSIS), Vol. 16, No. 2, pp 56-62.

[3] Burney, S. M Aqil, Nadeem Mehmood, 2012 "Generic Temporal and Fuzzy Ontological Framework, (GTFOF) for Developing TemporalFuzzy Database Model for Managing Patient's Data, Journal of Universal Computer Science, vol. 18, no. 2 (2012), 177-193.

[4] Canda, K.Selcuk, "Resource Description Frame work: Metadata and Its Applications", Arizona State University, http://citeseerx.ist.psu.edu

[5] Connor, Martin O et al , "Querying the Semantic Web with SWRL",
Stanford Medical Informatics, Stanford University School of Medicine, Stanford, CA 94305 martin. oconnor @stanford.edu.

[6] Darai, D.S S Singh, S Biswas, 2010, "Knowledge Engineering an overview", International Journal of Computer Science and Information Technologies, Vol. 1 (4), 230-234.

[7] Hoogenboom, G P.W. Wilkens, P.K. Thornton, et al., 1999. "Decision support system for agro technology transfer" v3.5. In: DSSAT version 3, vol. 4 University of Hawaii, Honolulu, HI, pp. 1-36.

[8] Kalibatiene, Diana et al , 2010, “Ontology-Based Application for Domain Rules Development" Scientific Paper University of Latvia, Vol 756, Computer Science and Information Technologies pp 9-32.

[9] Khan, Ghanzafar, Ali, 2010, "Present and prospective role of electronic media in the dissemination of agricultural technologies among farmers of the Punjab, Pakistan, Ph.d theses, university of Agriculture Faisalabad, Pakistan.

[10] Kolhe Savita, Raj Kamal, Harvinder S. Saini, G.K. Gupta, 2011, “A web-based intelligent disease-diagnosis system using a new fuzzy-logic based approach for drawing the inferences in crops", Computers and Electronics in Agriculture Volume 76, Issue 1, Pages 16-27.

[11] Magarey, R.D.; Travis, J.W.; Russo, J.M.; Seem, R.C. \& Magarey, P.A. 2002. Decision Support Systems: Quenching the Thirst. Plant Disease, Vol. 86, No. 1, pp. 4-14,

[12] Naseem, Jawed, S. M Aqil Burney, 2018, "Decision Making in Uncertainty: A Bayesian Network for Plant Disease Diagnoses" International Journal of Computer Science and Information Security (IJCSIS), Vol. 17, No. 2, February 2018.

[13] Natalya F. Noy and Deborah L. McGuinness, "Ontology Development 101: A Guide to Creating Your First Ontology” Stanford University.

[14] Zheng, Weiguo et al, "Semantic SPARQL Similarity Search Over RDF Knowledge Graphs", http://www.vldb.org/pvldb/vo19/p840-zheng.pdf, pp 840-851.

[15] Zhi Ping Ding, 2011, "The Development of Ontology Information System Based on Bayesian Network and Learning," Advances in Intelligent and Soft Computing", Volume 129, , Pages 401-406. 